ÉGYPTE

monde arabe

\section{Égypte/Monde arabe}

$34 \mid 1998$

Droits d'Égypte : histoire et sociologie

\title{
Entretien avec le conseiller Yahyâ al-Rifâ‘î
}

Entretien conduit par Muhammad 'Abd al-Quddûs pour alSha'b(14 octobre 1997)

Traducteur : Baudouin Dupret

\section{OpenEdition}

\section{Journals}

Édition électronique

URL : https://journals.openedition.org/ema/1529

DOI : 10.4000/ema.1529

ISSN : 2090-7273

Éditeur

CEDEJ - Centre d'études et de documentation économiques juridiques et sociales

Édition imprimée

Date de publication : 31 décembre 1998

Pagination : 203-213

ISSN : 1110-5097

Référence électronique

"Entretien avec le conseiller Yahyâ al-Rifâî̀ », Égypte/Monde arabe [En ligne], 34 | 1998, mis en ligne le 11 juillet 2008, consulté le 07 juillet 2022. URL : http://journals.openedition.org/ema/1529 ; DOI :

https://doi.org/10.4000/ema.1529

Ce document a été généré automatiquement le 7 juillet 2022.

Tous droits réservés 


\section{Entretien avec le conseiller Yahyâ al-Rifầî}

Entretien conduit par Muhammad 'Abd al-Quddûs pour alSha'b(14 octobre 1997)

Traduction : Baudouin Dupret

1 Demain commence le procès crucial de six de nos confrères du journal al-Sha'b inculpés pour outrage (ihâna) au ministre de l'Intérieur, le général Hasan al-Alfî, que notre journal a accusé de corruption financière, outre sa corruption politique qui se traduit dans le trucage des élections et les atteintes aux droits de l'homme.

2 Nous espérons de notre procès qu'il rende justice, ce qui n'est pas simple. Les tribunaux exercent le pouvoir judiciaire, qui peut être utilisé aussi bien pour la justice ou l'oppression. Entre les mains d'un gouvernement juste, c'est le plus formidable moyen d'implantation de la justice et du droit, mais entre les mains d'un gouvernement inique, c'est le plus formidable outil de répression et d'anéantissement du droit et de toute réforme. Ce ne sont pas mes propos, mais ceux de Ahmad Fathî Surûr, Président de l'Assemblée du Peuple, dans son commentaire du Code pénal, qui les tenait de l'un des chefs de file de la renaissance islamique indienne.

3 Dans ce qui suit, on trouvera un entretien avec l'un des grands juristes du pays, le conseiller al-Rifâ'î, qui explique comment le régime interfère dans les affaires judiciaires et tente d'influencer le cours de la justice.

\section{Présentation du conseiller Yahyâ al-Rifâ'î}

4 La justice se renforce si le judiciaire est indépendant, mais ceci est menacé dans notre pays, en dépit de la Constitution qui le confirme. En Égypte, le régime, depuis bien longtemps, s'ingénie à interférer de différentes façons dans les affaires judiciaires.

5 J'ai choisi pour discuter de ce sujet le conseiller Yahyâ al-Rifâ'î, ancien vice-président de la Cour de cassation, président du premier Congrès de la justice (tenu en 1986), plusieurs fois élu par les juges égyptiens à la présidence de leur club, dont il est maintenant président à vie. Cet homme est doté d'une personnalité exceptionnelle. Il a 
passé sa vie au service de la justice et de la promotion des droits de l'homme en Égypte. Il a remporté pour cela le prix de feu Fathî Radwân. À l'époque de Nasser, il a participé à la déclaration du Club des juges, en mars 1968, dans laquelle les membres du pouvoir judiciaire donnèrent leur avis sur les causes de la débâcle de 1967, dont la plus importante était l'absence des droits et libertés fondamentaux des citoyens. Ils demandèrent que les juges restent à l'écart des organisations de l'Union socialiste et c'est pour cela que le conseiller Yahyâ al-Rifâ'î fit partie des 129 juges que le « Massacre de la magistrature » (madhbahat al-qadâ) frappa en août 1969.

6 À l'époque de Sadate, il contribua, par sa position au Club des juges, à exercer une pression sur le gouvernement pour qu'il retire son projet de loi « Protection des valeurs contre la honte» (himâyat al-qiyam min al-'ayb), un projet controversé que les différentes forces de la Nation avaient rejeté. Cela signifiait la fin de sa nomination à la Cour de cassation et il obtint que la Cour prononce un arrêt individuel pour l'y nommer et qu'elle réponde ainsi positivement à quatre décisions des assemblées générales qui s'y étaient montrées favorables.

7 Il organisa, à l'époque du président Moubarak, alors qu'il était à la présidence du Club des juges, le premier (et dernier) Congrès de la justice d'avril 1986. Son discours devant le président de la République fut considéré, à cette occasion, comme un manifeste de défense des droits de l'homme égyptien. Voilà quelques-unes des réalisations du conseiller Yahyâ al-Rifâ'î, qui a accepté sans hésitation de s'entretenir avec moi d'un sujet épineux et sensible: les embûches de la législation et de son application qui menacent l'indépendance des juges en Égypte.

\section{Al-Sha'b : Pourquoi ne parle-t-on pas d'indépendance des juges dans les pays développés ?}

Yahyâ al-Rifâ'î: L'indépendance et la garantie de l'indépendance du judiciaire sont des principes dont on ne parle pas dans les vraies démocraties du monde développé, telles que la Grande-Bretagne ou l'Allemagne, parce qu'il s'agit de principes inscrits de manière vraiment constante dans la conscience des peuples et des dirigeants de ces États. Ils savent que ce sont là des valeurs humaines essentielles pour la construction de l'État, à l'image de valeurs comme le dévouement chez le médecin, le savoir chez l'enseignant ou la probité chez le notaire. Si l'un de ces derniers vient à manquer de ces qualités, il perd toute légitimité. Il en va de même pour cette valeur qu'est l'indépendance et la garantie de l'indépendance du judiciaire. Si elle n'est pas vraiment mise en œuvre, le judiciaire perd toute valeur, de même que les droits et libertés des citoyens, qui ne jouissent plus alors de véritable protection. Il est de la nature du judiciaire d'en aller ainsi. Tout ce qui touche à cette indépendance fait pencher la balance de la justice, ouvre grand les portes de la corruption, fait place à l'oppression et conduit les structures du gouvernement à la ruine.

Tous ces principes évidents continuent à n'être que de simples slogans dont se moquent les gouvernants des démocraties toutes théoriques du tiers-monde où le pouvoir exécutif domine les pouvoirs législatif et judiciaire, au point qu'il façonne le pouvoir législatif selon son vouloir et n'éprouve aucune gêne à ce que les élections soient truquées sous ses propres yeux, voire sciemment, pour qu'une majorité d'au moins deux tiers soit assurée et détienne le dernier mot dans tous les domaines de compétence de ce pouvoir.

Il interfère aussi avec le pouvoir judiciaire et réduit son indépendance, que ce soit en élargissant les compétences de la justice militaire ou des autres types de justice 
exceptionnelle, en réduisant les droits des citoyens à aller en justice ou en portant atteinte aux garanties d'indépendance des juges, voire encore en assurant toutes les garanties législatives nécessaires pour qu'aucune décision ou disposition du Parquet général - ou même aucun jugement des tribunaux - ne soit prise dans les questions qui touchent aux intérêts du pouvoir exécutif ou de ses représentants.

Al-Sha'b : Quelles sont ces garanties pernicieuses?

Y. al-Rifâ'î: [La garantie d'indépendance], par exemple, puisque le Parquet général lui est subordonné et qu'il est seul habilité à choisir le Procureur général et à le nommer, sans entrave ni condition. Il garde également pour lui-même le pouvoir de définir et de modifier les salaires et avantages financiers des magistrats, jusqu'à les mettre dans la position de les mendier. Il se réserve la mainmise sur l'appareil administratif qui contrôle le travail des magistrats, évalue leur compétence, leur aptitude et leur qualification à continuer d'exercer leurs fonctions, décide leur mutation, leur affectation et leur détachement et leur offre, ainsi qu'à leur famille, les services sanitaires et sociaux.

Dans ces pays [pseudo-démocratiques], le pouvoir exécutif adjuge à certains de ceux qui occupent des positions dirigeantes dans le Parquet général ou dans la magistrature des avantages matériels et financiers en contravention à la Constitution et à la loi, comme il alloue à certains d'entre eux une voiture gouvernementale - et parfois plusieurs - ou de généreuses primes en liquide, par des voies détournées ou non et sans justification apparente, jusqu'à rémunérer certaines personnes en échange de leur présence à l'audience, alors que c'est de la nature même de leur travail, ou payer des sommes considérables à d'autres pour qu'ils attestent de la nécessité de rémunérer un garde qui leur soit affecté personnellement. Tout cela vise à dresser les magistrats à lui obéir et à veiller à son bien, de sorte qu'en fin de compte, le judiciaire devient le protecteur du pouvoir exécutif et non le protecteur des droits, des libertés et de la justice. C'est pour cela qu'on dit que le juge qui accomplit sa mission à l'ombre d'un pouvoir inique est dans une position peu enviable. Un rapport récent de la Commission des droits de l'homme des Nations unies a répertorié de nombreux exemples de ces manquements, et d'autres aussi, qui portent souvent atteinte, dans les pays du tiers-monde, aux intérêts des gens, si bien que la confiance dans la justice en sort ébranlée.

\section{Al-Sha'b : La confiance dans la justice et les juges est-elle affectée par ces procédés ?}

Y. al-Rifâ'î. - Il ne suffit pas de dire que, dans la Constitution, le pouvoir judiciaire est indépendant, mais il faut aussi que les gens aient confiance dans le fait qu'on ne peut influencer leur juge.

\section{La situation en Égypte}

Al-Sha'b: Quelle est la situation de l'indépendance du judiciaire et de ses garanties chez nous?

Y.al-Rifâ'î: Le principe de l'indépendance du judiciaire est établi chez nous par les textes de la Constitution, ainsi que d'autres. Il se traduit également dans la loi sur le pouvoir judiciaire.

Notre Constitution stipule que "l'indépendance et l'inviolabilité du judiciaire constituent deux garanties fondamentales dans la protection des droits et libertés; 
que le pouvoir judiciaire est indépendant, que les tribunaux, dans leur variété et leurs différents degrés, en ont la responsabilité et qu'ils prononcent leurs jugements suivant la loi [...] ; que les juges sont libres, qu'il n'y a pas de pouvoir autre que la loi qui s'exerce sur eux dans l'exercice de la justice et qu'aucun pouvoir n'est autorisé à interférer dans leurs affaires ou dans les affaires de la justice ».

La première garantie véritable de l'indépendance du judiciaire réside toutefois chez nous dans la conscience morale et la vertu propre des juges. Ils ont prouvé, grâce à Dieu, au fil des années, que, dans leur écrasante majorité, ils étaient de taille à justifier cette confiance, en dépit de l'avalanche des textes législatifs, des pratiques anormales et des pressions contraires à ce que signifie et implique le principe d'indépendance du judiciaire. C'est comme si on n'avait besoin de ce principe que dans les situations difficiles et qu'autrement il ne s'agissait que d'un slogan vide de contenu. Les juges occupent alors la position de commandos, dans la mesure où les textes qui établissent les garanties d'indépendance du judiciaire n'ont pour fonction que de faire face aux temps difficiles et qu'en dépit de cela, le pouvoir exécutif n'en fait bon marché que dans ces moments-là.

\section{Textes et pratiques contraires à la constitution et aux lois}

\section{Al-Sha'b : Quelles sont ces textes et pratiques?}

Y. al-Rifâ'î: Il y a le texte figurant dans la loi sur le pouvoir judiciaire et qui concerne la soumission des membres du Parquet à leurs présidents et ensuite au ministre de la Justice. C'est une chaîne logique dans un appareil administratif, mais elle n'est pas admissible dans l'organisation du pouvoir judiciaire, parce qu'elle porte atteinte à l'indépendance obligatoire des membres du Parquet en leur qualité de personnes investies des pouvoirs du juge d'instruction et non pas seulement de personnes disposant du pouvoir d'inculper.

Il y a aussi le texte figurant dans cette loi sur la compétence exclusive du pouvoir exécutif dans le choix et la nomination du Procureur général, sans qu'il n'y ait pour condition l'accord du Conseil supérieur de la magistrature, ni que ce ne soit limité par les règles d'ancienneté, de qualification et d'aptitude, ni même que ne soit exigé qu'il ait siégé au cours de sa vie judiciaire pendant une période suffisante pour avoir autorité sur le judiciaire. Du fait qu'il est porté atteinte à toutes ces conditions, la porte est ouverte à ce que le développement de cette fonction soit davantage prisonnier de considérations et d'ambitions personnelles qui impliquent sa soumission au pouvoir ayant autorité pour le nommer, quand bien même ce serait au détriment des droits et des garanties des individus en général et des opposants en particulier.

Parmi les textes contraires à la Constitution, il y a également le texte dans la loimême qui organise au sein du ministère de la Justice une administration d'inspection judiciaire du travail des magistrats, qui a compétence pour organiser la mobilité judiciaire avec ce que cela comporte en matière de promotion, de planification, de mutation et nomination des membres du judiciaire, ainsi que de vérification et d'évaluation de leur travail, voire même d'enquête à leur sujet pour tout ce qui les concerne et pour toutes les plaintes et rapports qui sont présentés contré eux ou 
encore d'introduction des plaintes disciplinaires et des plaintes portant sur l'aptitude. Il y a aussi la compétence du ministre à notifier aux juges et aux présidents de tribunaux des degrés de leur compétence et à enjoindre au Ministère de donner priorité à la promotion de ceux qui ont sa préférence. Et cela sans tenir compte de toutes les compétences dont ce ministère et le ministre, en tant qu'organes du pouvoir exécutif, disposent effectivement dans le domaine des pouvoirs et de la carrière des juges et des membres du Parquet général - matières qui échappent au contrôle du Conseil d'État ou de la Haute Cour constitutionnelle -, ainsi que de leur capacité à intimider les magistrats et à porter atteinte à leur indépendance qui est pourtant garantie par la Constitution. La situation ne se trouve nullement affectée par le fait que les membres de cette administration sont des magistrats détachés, parce qu'ils appartiennent dès ce moment au pouvoir exécutif, outre qu'ils tirent de cela quelques avantages exceptionnels qui rendent ce détachement réellement avantageux.

\section{Al-Sha'b : Quelles répercussions cela a-t-il sur les intérêts des justiciables ?}

Y. al-Rifâ'î: Je n'insisterai pas sur le danger que tout ceci représente pour les intérêts des justiciables. Cette administration a entrepris de publier, au cours des dernières années - et avec l'accord du ministre bien sûr - des circulaires à destination des magistrats qui traitent de l'accomplissement de leur mission. Cela, elle l'avait entrepris à l'époque de la domination anglaise, mais l'Égypte y avait renoncé depuis des dizaines d'années, pour ne pas porter atteinte à l'indépendance des magistrats et au principe qui interdit de les soumettre à une orientation quelconque, d'où et de qui elle puisse venir.

Le plus fort, dans tout cela, c'est que le ministère n'a pas éprouvé la moindre gêne à prendre ses libertés par rapport aux années précédentes. Une de ces circulaires s'attache même à mettre en garde les présidents de tribunal et les magistrats contre toute apparition dans la presse qui traite des affaires concernant les personnalités exerçant des responsabilités importantes, et ce en contradiction avec le règlement d'inspection judiciaire lui-même. Naturellement, la volonté persistante de systématiquement mettre en œuvre cette circulaire dans les tribunaux a eu un effet d'intimidation sur le juge quand il s'est agi de parler dans le cadre d'affaires de ce type. Il a un intérêt évident à éviter que le pouvoir exécutif ne se mette en colère contre lui. Le besoin est pourtant pressant de croire à sa vertu, à sa neutralité et à son indépendance plutôt que de lui porter atteinte gravement en ébranlant la confiance générale dans la justice et les juges, voire en discréditant les jugements eux-mêmes, même si aucun juge n'y a vraiment pris part.

Parmi ces textes contraires à la Constitution, il y a également celui qui donne le pouvoir ultime de choisir et d'affecter les conseillers à la présidence des tribunaux de première instance - ainsi que de mettre fin à cette affectation ou à son renouvellement - au seul ministre de la Justice, sans qu'il ait besoin de l'accord du Haut Conseil de la magistrature. Ceci rend ces conseillers administrativement dépendants du ministre, alors même qu'ils sont à la tête des juges du tribunal et que, plus important encore, ils exercent une compétence judiciaire dans le jugement de ces derniers, qu'ils ont le droit de sélectionner les affaires et de prononcer des injonctions judiciaires, qu'ils peuvent modifier la répartition du travail à n'importe quel moment, en cas de doute sur la confiance qui peut leur être faite, pour attribuer ces affaires à des juges qu'ils ont choisis, dans les cas de récusation par exemple. Il y a 
enfin ces compétences qui ont été ajoutées à celles que détenaient déjà ces conseillers dans le domaine des élections syndicales et autres. C'est sans précédent et fondamentalement hors de leur pouvoir ; cela ouvre de plus la porte à la mise en cause de leurs attitudes dans ce cadre, ce qui n'est pas sans répercussion sur la confiance qu'on peut avoir en leur indépendance, surtout après que certains ont été nommés à des fonctions politiques.

En dépit du texte établi par la loi qui stipule que «les salaires des magistrats et des membres du Parquet sont fixés d'après le barème annexé à la loi et [qu'] il ne peut être octroyé à aucun d'eux une rémunération personnelle ni un traitement exceptionnel sous quelque forme que ce soit ", il est de pratique constante que le ministère contrevienne lourdement à ces règles. Il oublie d'augmenter le montant des pensions et de répondre à l'effondrement du pouvoir d'achat des allocations et salaires stipulés dans ce barème depuis plus de trente ans. Quand la situation s'est aggravée et quand les juges et leurs représentants ont sollicité de manière pressante leur révision, il a entrepris de payer ce qu'on appelle des incitants, qui permettent en soi d'en exclure certains, et il a inventé le système des séances du soir, des séances spéciales, etc. Il a maintenu les magistrats au travail pendant les vacances judiciaires, en contradiction avec la loi elle-même. On trouve encore d'autres formes de discrimination et d'expédients, sur le plan de l'octroi aux magistrats et aux membres du Parquet de certains de leurs droits légitimes, dans l'obtention des allocations financières permettant de faire face à l'augmentation du coût de la vie et prenant la forme de paiements complémentaires ou d'indemnités. Tout ceci, alors que l'Etat devrait impérativement modifier de lui-même le barème des salaires et aligner cette modification sur le pouvoir d'achat et sur ce qui est nécessaire pour préserver l'honneur et la responsabilité qui sont liés à la fonction des juges, ainsi que le dit la Déclaration internationale sur l'indépendance de la justice.

Le ministère de la Justice n'a donc pas éprouvé de gêne à favoriser certains en leur réservant un traitement exceptionnel et en en privant les autres, que ce soit en établissant et en mettant en œuvre les règles et les délais des prêts ou en répartissant les séances d'arbitrage, les gains illégaux, les permanences du Parquet général quand elles entraînent des gains exceptionnels. Tout ceci contrevient aux exigences de la loi sur le pouvoir judiciaire qui interdit d'établir une discrimination entre ses membres et de décider tout traitement exceptionnel en faveur de l'un d'eux, quelle qu'en soit la forme.

\section{La malversation du système électoral}

Al-Sha'b: Cela a-t-il des répercussions sur le rôle des magistrats dans la supervision des élections générales?

Y. al-Rifâ'î : Le pouvoir exécutif a continué à charger les magistrats, à chaque élection générale, de la supervision, de l'annonce des résultats et de la garantie de l'impartialité de ces élections - alors même que cette supervision n'est pas effective -, pour ensuite compenser le malaise des magistrats en décidant de leur payer des indemnités : quelques centaines de livres pour chaque magistrat ayant participé à la supervision, pour tout ou partie d'une journée, et même plusieurs milliers de livres pour tous ceux qui ont supervisé ces derniers, qu'ils soient conseillers, présidents de tribunal ou inspecteurs judiciaires, sans même solliciter l'accord du Haut Conseil de 
la magistrature au sujet de ces indemnités, versées par la police. On rapporte même de source sûre que les magistrats du tribunal de Béni Soueif ont, d'un commun accord, renvoyé officiellement en un seul chèque au ministère de l'Intérieur cet argent que chacun d'eux avait eu la surprise de recevoir de la police sous le manteau, en indemnité de cette supervision.

De la même manière, le pouvoir exécutif garde pour lui le contrôle de la Caisse des services sociaux et de santé des membres de la magistrature et du Parquet général, ce qui lui permet de jouer avec le paiement des soins de santé en dehors des hôpitaux avec lesquels la Caisse a passé un contrat d'opérer des discriminations entre les ayants droit, d'en exclure certains sans la moindre considération pour les règles constitutionnelles, ou encore de faire des distinctions pour ce qui est des soins à l'étranger.

Al-Sha'b: Ya-t-il eu dans notre histoire judiciaire des mécanismes de limitation de ces pratiques et manœuvres contraires à la Constitution et à la loi ?

Y. al-Rifâ'î: Oui, nous avons un modèle éclatant auquel l'Égypte a refusé de donner suite depuis plus de 70 ans, quand il fut décidé d'octroyer trois gratifications à un président, un substitut et un conseiller de la cour d'appel. Sa'ad Zaghlûl et Fakhrî 'Abd al-Nûr ont marqué leur opposition à cette distinction, lors de la session de l'Assemblée des députés du 10 août 1926. Sa'ad a déclaré : « La magistrature doit être indépendante et elle ne peut être indépendante qu'à condition que le pouvoir exécutif ne s'en mêle pas. L'organisation de la magistrature en Égypte facilite l'immixtion du pouvoir exécutif par des voies légales - et il faut s'en désoler -, mais il s'y immisce également par des voies illégales, voire même par la corruption de la magistrature. Cela n'est pas tolérable. Il vous faut, en votre qualité de députés de la Nation, frapper toute main qui toucherait à cette indépendance ". L'Assemblée a décidé ce jour-là «de rayer ces gratifications du budget, d'exclure toute atteinte à l'indépendance de la magistrature et d'établir le principe interdisant d'octroyer à un magistrat des gratifications personnelles ». Tout ceci est établi dans les comptes rendus du Sénat, en vertu du texte de la Constitution, édition de l'année 1940, page 2610.

Al-Sha'b : Quel chemin faudrait-il suivre selon vous pour sortir de cette impasse?

Y.al-Rifâ'î: Le mémoire additionnel à la première loi sur l'indépendance de la magistrature, promulgué en 1943, a affirmé que cette loi ne constituait qu'une simple étape sur le long chemin de l'indépendance, mais les gouvernements qui se sont succédé par la suite ont passé de nombreuses législations qui étaient des régressions par rapport à la première étape. L'Égypte n'a pas progressé sur cette voie, mais elle a au contraire reculé et trébuché, jusqu'au moment où la magistrature est devenue prisonnière de tous ces textes et pratiques honteux qui violent son indépendance et les garanties qui lui sont apportées. Au point qu'il s'est justifié d'en faire le sujet d'un des comités principaux du premier Congrès de la justice que les magistrats ont tenu au Palais de Justice du 24 au 28 avril 1986. Le Congrès a alors recommandé qu'on inscrive dans la Constitution les règles fondamentales d'organisation des questions concernant la magistrature, protégeant ainsi sa stabilité et la préservant du changement ; qu'on laisse au Conseil supérieur de la magistrature le soin d'établir le détail des règles, les amendements et le contrôle de leur mise en œuvre; que les magistrats et les membres du Parquet général soient indépendants pour ce qui touche à la gestion de leurs affaires administratives - en attribuant au Conseil 
supérieur de la magistrature la compétence de préparer le budget de la magistrature, de sorte qu'y soit défini ce qui lui est alloué et ce qu'elle dépense ; que soit inséré un article unique dans le budget général de l'État, comme c'est le cas pour le pouvoir législatif et d'autres encore comme la Haute Cour constitutionnelle, l'Administration centrale des comptes et l'Administration du procureur socialiste, de sorte que le pouvoir exécutif n'arbitre pas la question des besoins de la magistrature et des magistrats, ce qui implique nécessairement que la détermination et la modification des salaires, des compétences et des pensions des magistrats, des membres du Parquet et de leurs auxiliaires appartienne au Conseil supérieur ; qu'il soit mis fin à la sujétion de l'administration de l'inspection judiciaire au ministère et qu'elle soit rattachée avec toutes ses compétences au Conseil supérieur de la magistrature, comme cela a été effectivement établi par le Conseil d'État; que soit établie, même progressivement, la distinction entre les pouvoirs d'enquête et d'inculpation ; qu'il soit également mis fin à la sujétion du Parquet général et de ses membres au ministre de la Justice, qu'il soit rattaché au Conseil supérieur et que soit élargi par la suite le système du Parquet civil pour qu'il remplisse son rôle en instruisant les requêtes à tous les niveaux de juridiction, si bien qu'il sera chargé du contrôle quotidien de la validité des procédures et de la publicité, qu'il y aura une véritable accélération dans la conduite de l'instruction et que les requêtes seront préparées de telle sorte qu'on puisse statuer dans le plus bref délai possible; que la loi assure les garanties suffisantes dans les questions de discipline et de compétence, aux deux niveaux; que les pouvoirs des membres du Parquet dans la supervision des prisons puissent être véritablement exercés; que soient renforcées les compétences du Conseil supérieur de la magistrature en établissant dans un texte que la nomination du président de la Cour de cassation, du Procureur général et des autres magistrats et membres du Parquet général, leur mutation, leur détachement et leur affectation ne peuvent intervenir qu'après consentement du Conseil et en fonction de règles générales claires et établies qu'on ne peut pas enfreindre et auxquelles il ne peut être dérogé, que soit renforcé le Centre d'études judiciaires pour lui permettre de former les magistrats de tous niveaux et de toutes compétences, qu'il soit évidemment rattaché au Conseil et que soit définie l'étendue de l'action du Parquet général et l'étendue de la délégation des fonctions de la magistrature, tout en supprimant définitivement toute délégation à autrui pour préserver la dignité du juge et son prestige et pour qu'il puisse entièrement se consacrer à son seul travail ; que la gestion des affaires d'assistance médicale et sociale aux magistrats et à leurs familles soit confiée à un conseil qui émane d'eux, sans que le pouvoir exécutif ne le contrôle ou n'y participe ; que soient adjoints dans la composition du Conseil supérieur de la magistrature des conseillers de la Cour de cassation et de la cour d'appel du Caire qui seraient choisis par l'assemblée générale de chacune de ces deux cours, afin de renforcer la composition du Conseil et promouvoir le travail qui lui a été confié depuis la création du premier Conseil supérieur de la magistrature en Égypte, jusqu'à ce que la présence de membres élus dans la composition du Conseil permette effectivement la réforme des affaires de la magistrature et des magistrats.

Al-Sha'b : À quoi ont abouti ces recommandations?

Y. al-Rifâ'î: Je peux dire malheureusement que le gouvernement n'a pas mis en œuvre la moindre de ces propositions, si bien que les magistrats ont alors formé un comité regroupant les présidents de la Cour de cassation et les principaux magistrats, qui a rédigé un projet de loi de mise en œuvre de la plupart de ces propositions, à la 
lumière de l'information mondialement disponible sur l'indépendance de la magistrature. Ce projet a été publié par l'assemblée générale du Club des juges dès 1991, et il comprend de nombreuses réformes du régime de la justice qui préservent les intérêts des justiciables et renforcent la confiance générale dans la justice et les magistrats en les mettant vraiment à l'abri de toute influence. Le gouvernement a cependant ignoré ce projet et a même accentué les défaillances du système au cours de ces dernières années en se lançant dans tout ce dont nous avons déjà parlé : il a multiplié les pratiques illégales et inconstitutionnelles, il a porté atteinte aux intérêts des justiciables, il a ébranlé la confiance générale et a motivé la rumeur, et plus particulièrement à chaque fois qu'un jugement de mise sous tutelle d'un syndicat professionnel a été prononcé, en bafouant la parole de la Cour de cassation dont l'autorité s'élève pourtant au-dessus de la loi, ou encore chaque fois que ces infractions et ces pratiques fautives expliquent qu'un criminel scélérat ose s'en prendre à l'honneur de la grande magistrature égyptienne. Mais les juges - et tous ceux qui sont attachés à l'indépendance des juges et de la justice en Égypte - ne cessent de garder l'espoir de voir le gouvernement changer de conduite et ils ont posé les premiers jalons de la réforme légale dont on a parlé. Ils ont de la sorte ouvert les portes de la réforme et du progrès du pouvoir judiciaire, donnant ainsi les garanties les plus importantes aux citoyens et implantant les règles de la vraie justice dans le pays.

Pour terminer, je vous répète qu'il ne suffit pas que la Constitution dise que le judiciaire est un pouvoir indépendant et qu'il est interdit de s'immiscer dans ses affaires, mais qu'il faut aussi que les gens aient confiance dans le fait que leurs juges ne sont effectivement pas soumis à une influence quelconque.

INDEX

Mots-clés : droit, Frères musulmans, justice, islam et politique, islamisme, politique 